\title{
Ambulance Siren Noise Reduction using LMS and FXLMS Algorithms
}

\author{
Manoj Kumar Sharma ${ }^{1 *}$ and Renu Vig ${ }^{2}$ \\ 'Department of Electrical and Electronics Engineering, UIET, Panjab University, Sector 14, \\ Chandigarh, 160014,India; mks_uiet@pu.ac.in \\ ${ }^{2}$ Department of Electronics and Communication Engineering, UIET, Panjab University, Sector 14, \\ Chandigarh, 160014, India; renuvig@hotmail.com
}

\begin{abstract}
Objectives: In case of medical emergency, an ambulance uses siren (which produces noise up to $120 \mathrm{~dB}$ ) to alert the traffic on the road to give way. However, siren noise is troublesome to the patient lying inside. This calls for noise control inside the ambulance. Methods/Statistical Analysis: The passive noise control methods are not feasible and economically inviable so active noise control (ANC) method is applied. The ANC is electro-acoustic technique in which the noise signal is acquired and noise with opposite phase is produced and overlapped on input noise to suppress the same. The weights are updated using adaptive algorithms so as to reduce the error in ANC system. In present work, least mean square (LMS) and filtered- reference least mean square (FxLMS) algorithm based ANC systems are applied and compared for ambulance siren noise reduction. MATLAB is used for simulating the ANC systems. Findings: Both the algorithms are compared on the basis of residual noise and smoothened ensemble average square error (SEASE). From the results, it is found that both algorithms LMS and FxLMS are good in noise reduction. The noise reduction for FxLMS based ANC system is $28 \mathrm{~dB}$ whereas it is $25 \mathrm{~dB}$ for LMS based ANC system, which indicates the superiority of FxLMS algorithm over LMS algorithm. The results are obtained analytically as well as indicated by power spectral density (PSD) plots of the residual noise. The comparison of SEASE for both algorithms also indicates better performance for FxLMS algorithm. The ANC system based on FxLMS algorithm is more stable as it considers the secondary path which includes practical components such as ADC, DAC, etc. Applications/Improvements: The results indicate that FxLMS algorithm based on ANC system can be implemented on the hardware using DSP processor. It can be further used for the other applications of noise reduction such as server noise, vacuum cleaner, etc.
\end{abstract}

Keywords: Active Noise Control, Ambulance Siren Noise, FxLMS Algorithm, LMS

\section{Introduction}

This paper explores a healthcare application of active noise control which is of utmost concern but never addressed. The application discussed is the reduction of acoustic noise generated by ambulance siren. The ambulance siren creates an acoustic noise up to $120 \mathrm{~dB}^{1}$. The ambulance serves the purpose of transporting patients in the critical condition to the hospital which must be done in minimum possible time. For this, the ambulance uses flashing light and siren, when it is on emergency run, to warn the people on the road for providing safe and quick passage. This is also mandatory in some countries where it is called the right of way, according to which the person in front has to pull his vehicle to make way for ambulance. However, the siren is helpful in one condition is a reason for the problem in

${ }^{*}$ Author for correspondence 
other. When siren noise is heard by the patient lying inside the ambulance, it results in anxiety and nervousness to him which will worsen his condition which is already critical and also, it can cause a loss of concentration to the ambulance driver. It is well known that patients are stressed by the noise generated by ambulance siren in the cabin during transport ${ }^{2-4}$. In the present work, active noise control technique is implemented through simulations using LMS and FxLMS algorithms and the results are compared for noise reduction of ambulance siren for the patient lying inside.

\section{Paper Outline}

In the section 2, the basic concept of the active noise control technique is described. The Least Mean Square (LMS) and Filtered Reference Least Mean Square (FxLMS) algorithm is discussed and their weight updating rule is specified. Section 3 covers the idea of use of active noise control in the ambulance. The simulation results are illustrated in section 4 and the conclusion is discussed in section 5 .

\section{Active Noise Control}

Active Noise Control technique is an electro-acoustic or electromechanical systems that eliminates noise signal and works on the principle of destructive interference. In active noise control an original signal is replicated by generating a signal which is exactly of the same amplitude but $180^{\circ}$ out of phase. This signal is known as anti-noise. The anti-noise when superimposed over the original noise signal results in a quieter region around the zone of interest $\frac{5-8}{}$. Active Noise Control (ANC) system provides better results than passive control techniques at low frequency and that too without any physical modifications. A conventional diagram of a single channel ANC system is presented in Figure $1^{?}$.

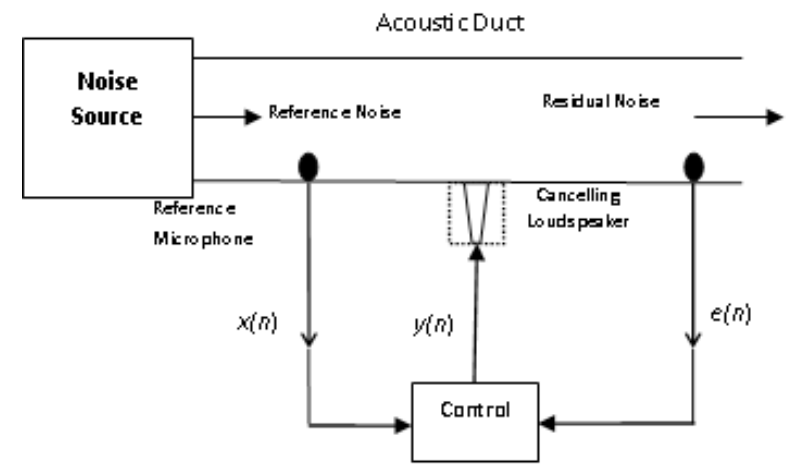

Figure 1. Conventional diagram of a single channel ANC system? 9
As depicted in Figure 1, the reference microphone catches the noise signal which is fed to the active noise control (ANC) system which consists of filters, ADC and DAC to create an anti-noise using LMS or FxLMS algorithm technique. The anti noise is fed back into region where noise has to be reduced through the cancelling loudspeaker. An error microphone is placed in the region of interest to receive the error signal which is fed back to the active noise control system to update the filter coefficients. Based on the error signal, the noise signal can further be replicated more efficiently so that error is further reduced.

\subsection{LMS Algorithm}

Least Mean Square (LMS) algorithm is one of the first algorithms used for noise cancellation. It is an adaptive FIR filter which estimates the desired filter such that least mean of square of error signal is obtained ${ }^{5,6}$. In this algorithm, the filter coefficients are adjusted to minimize the error. It is derived from the steepest descent method.

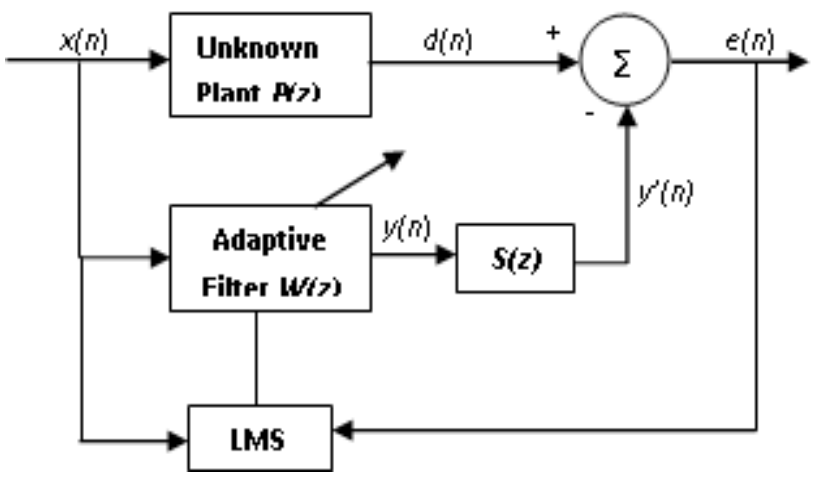

Figure 2. Block diagram of ANC using LMS Algorithm?

For LMS algorithm, the update equations for the filter coefficients are given (1) to (3),

$$
\begin{aligned}
& w(n+1)=w(n)+\mu \cdot x(n) \cdot e(n) \\
& e(n)=d(n)-y(n) \\
& y(n)=d(n)-w(n) x(n)
\end{aligned}
$$

where, $\mu \rightarrow$ learning rate (or convergence coefficient)

$e(n) \rightarrow$ error signal

$d(n) \rightarrow$ desired signal

$x(n) \rightarrow$ reference signal

$w(n) \rightarrow$ filter coefficients 


\subsection{FxLMS Algorithm}

The secondary path transfer function is $S(z)$ from $y(n)$ to $e(n)$ as shown in Figure 3. The lag in the path is introduced due to the control components such as ADC, DAC, filters, etc. which causes instability as it disrupts the alignment of the reference and error signal. This instability raises the need to estimate secondary path transfer function $S(z)$ as $S^{\wedge}(z)$, which modifies the LMS algorithm to Filtered reference LMS (FxLMS) algorithm. The estimation of $S(z)$ must be such that the phase error between $S(z)$ and $S^{\wedge}(z)$ must not be greater than $\pm 90^{\circ}$, otherwise the stability of the algorithm cannot be guaranteed ${ }^{10-12}$.

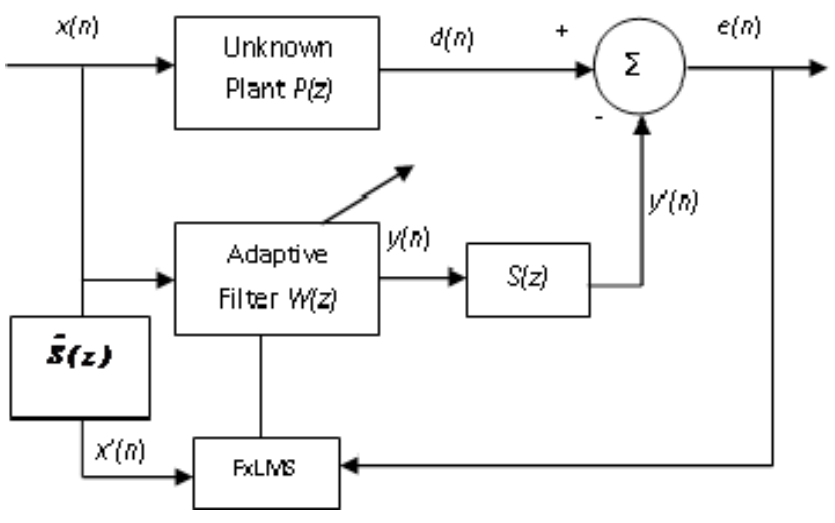

Figure 3. Block diagram of ANC using FXLMS Algorithm?․

The control signal is derived at any time ' $k$ ' by finite impulse response filtering operation as per (4),

$$
y(k)=\sum_{i=0}^{N-1} x(k-i) w_{i}(k)=x^{T}(k) w(k)
$$

filter weights updation is according to (5) and (6),

$$
\begin{aligned}
& \mathrm{w}(\mathrm{k}+1)=\mathrm{w}(\mathrm{k})+\mu \mathrm{e}(\mathrm{k}) \mathrm{x}^{\prime}(\mathrm{k}) \\
& \mathrm{x}^{\prime}(\mathrm{n})=\hat{\mathrm{s}}(\mathrm{n}) * \mathrm{x}(\mathrm{n})
\end{aligned}
$$

where, $\mu \rightarrow$ learning rate (or convergence coefficient)

$$
\begin{aligned}
& e(n) \rightarrow \text { error signal } \\
& d(n) \rightarrow \text { desired signal } \\
& x(n) \rightarrow \text { reference signal } \\
& w(n) \rightarrow \text { filter coefficients } \\
& x^{\prime}(n) \rightarrow \text { filtered reference signal vector } \\
& s^{\prime}(n) \rightarrow \text { impulse response of the secondary path }
\end{aligned}
$$
estimation filter, $S^{\wedge}(z)$
In ANC system, reference signal when passed through primary path is considered as primary disturbance $d(n)$, which is attenuated by acoustically superimposing antinoise signal, $y(n)$ as depicted in Figure 2. The path from $\mathrm{y}(\mathrm{n})$ to $\mathrm{e}(\mathrm{n})$ is called secondary path $\mathrm{S}(\mathrm{z})$ which accounts for the different electronics components required in hardware for processing of signals. The performance of electronics components such as ADC, DAC, amplifier etc. is varied, resulting in variation of $\mathrm{S}(\mathrm{z})$ which leads to instability of the system. To overcome the problem of instability in LMS algorithm due to introduction of secondary path, FxLMS algorithm is used ${ }^{13,14}$. In FxLMS algorithm, the reference is passed through estimated filter $S^{\wedge}(z)$, which is an estimate of $S(z)$ realizing its name as filtered reference. The filtered reference signal is then further used for updating the weights. Figure 3 illustrates the FxLMS based ANC system.

\section{Noise Control in Ambulance}

The noise generated from ambulance siren which is of a level of $120 \mathrm{~dB}^{2}$ penetrates the ambulance doors and reach the ears of the patient who is already in a critical condition and makes him restless. The noise generated can be stopped from entering the ambulance by use of physical barriers like installing sound absorbing material between doors but it will require a big amount of physical modification and also involves a considerable amount of money to be spent on the process. The patient can also put ear muffs to prevent noise from making him restless but considering his condition this solution is not feasible.

The solution based on active noise control (ANC) system can be used for this problem which is less costly than passive control and also without any physical modifications in the ambulance. In this solution, a local zone of silence is created around the head of patient making the noise to reduce to a considerable amount. This solution involves placing speaker, residual noise sensing microphones and a controller unit in the close proximity of the patient head without touching him. The controller unit consist of filters, ADC, DAC and a processor for processing the signal through an adaptive algorithm like LMS or FxLMS through digital signal processing.

This paper shows the noise reduction with ANC system in MATLAB using LMS and FxLMS algorithms and comparison between the results obtained from both the algorithms. 


\section{Simulations}

The simulations of the ANC for ambulance siren based on LMS and FxLMS algorithm are performed in MATLAB. The reference signal is obtained for 30 seconds from the ambulance. The ANC system shown in Figure 2 is simulated with measured $P(z)$ and $S(z)$. The filter length of 128 taps is taken primary $P(z)$ as well as secondary path $S(z)$. The learning rate, $\mu$, used is 0.04 for adaptive noise cancellation filter $W(z)$.

The input reference signal (ANC OFF) is shown in Figure 4 and the residual noise signal (ANC ON) after applying the ANC using LMS and FxLMS algorithm are shown in Figure 5 and Figure 6 respectively. It is observed that FxLMS algorithm is able to reduce the noise signal more effectively than LMS algorithm.

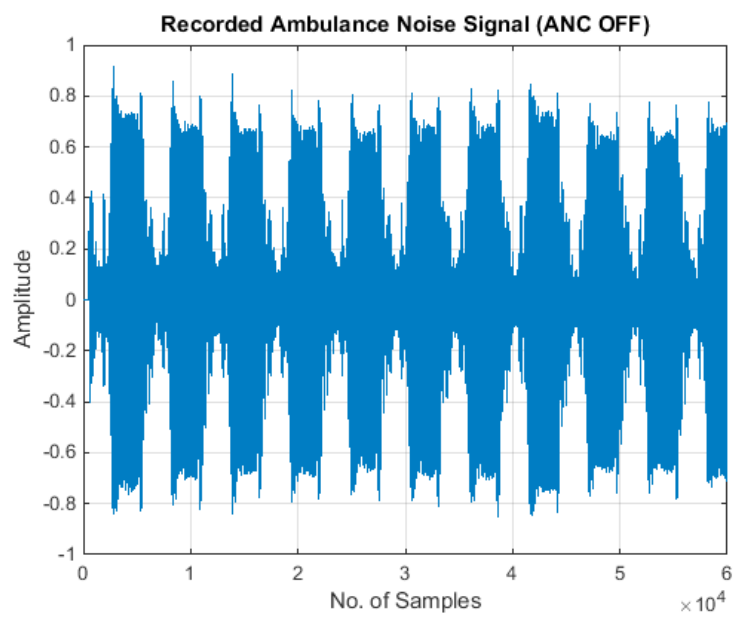

Figure 4. The input noise signal of ambulance siren.

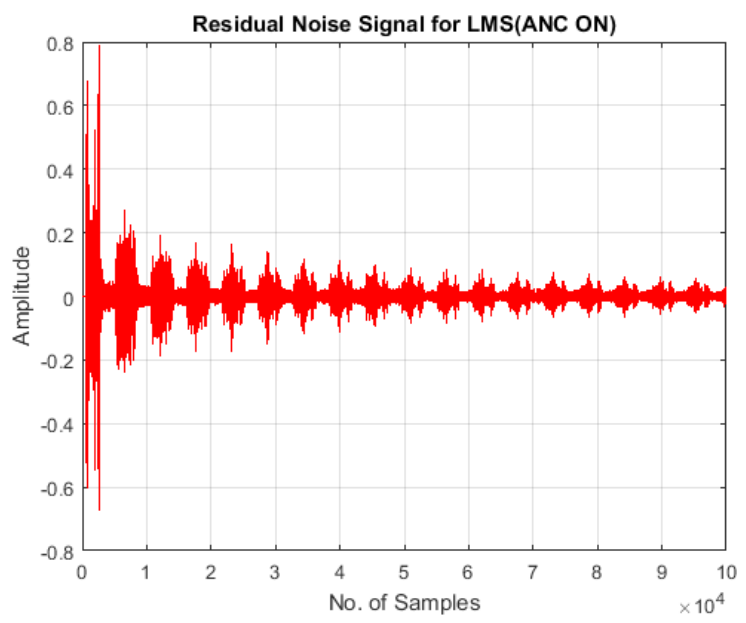

Figure 5. The Ambulance siren residual noise signal using LMS algorithm.

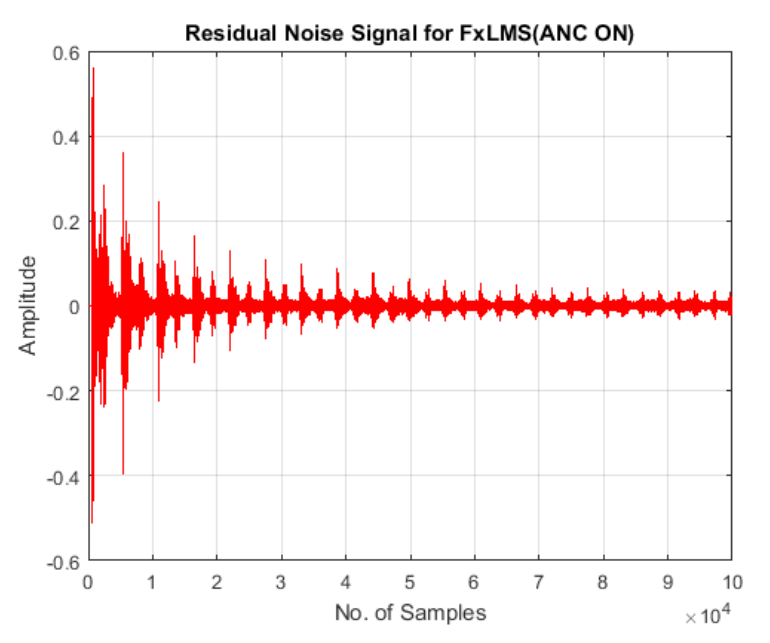

Figure 6. The Ambulance siren residual noise signal using FxLMS algorithm.

The signal to noise ratio is obtained by using the equation (7):

$S N R=10 \log _{10}\left(\frac{\sum d^{2}(n)\left(A N C_{-} O F F\right)}{\sum e^{2}(n)\left(A N C_{-} O N\right)}\right)$

The value of SNR comes out to be $24.8 \mathrm{~dB}$ for LMS algorithm and $28.1 \mathrm{~dB}$ for FxLMS algorithm, which indicates there is significant reduction in the noise signal using FxLMS algorithm. When the transients have decayed to zero, it is evident that a significant amount of noise reduction is achieved.

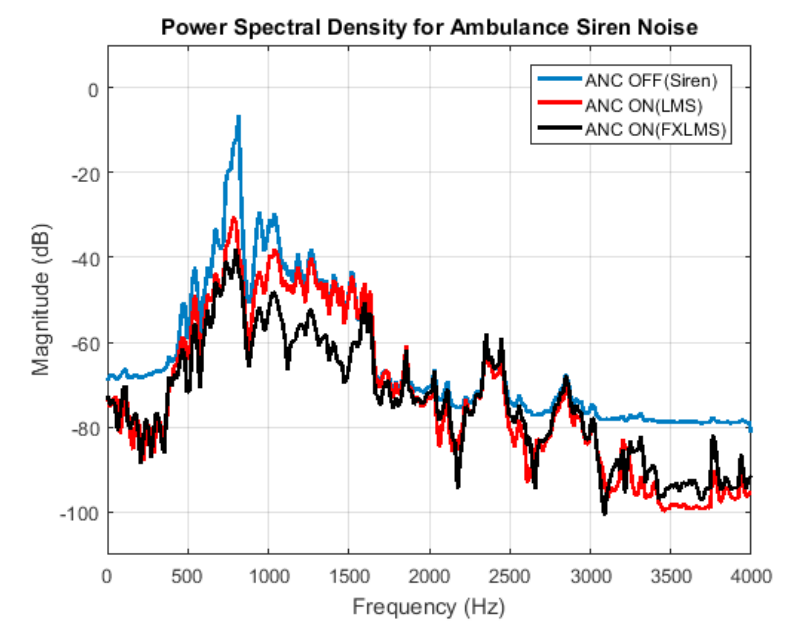

Figure 7. Power spectral density of reference and residual noise signal. 


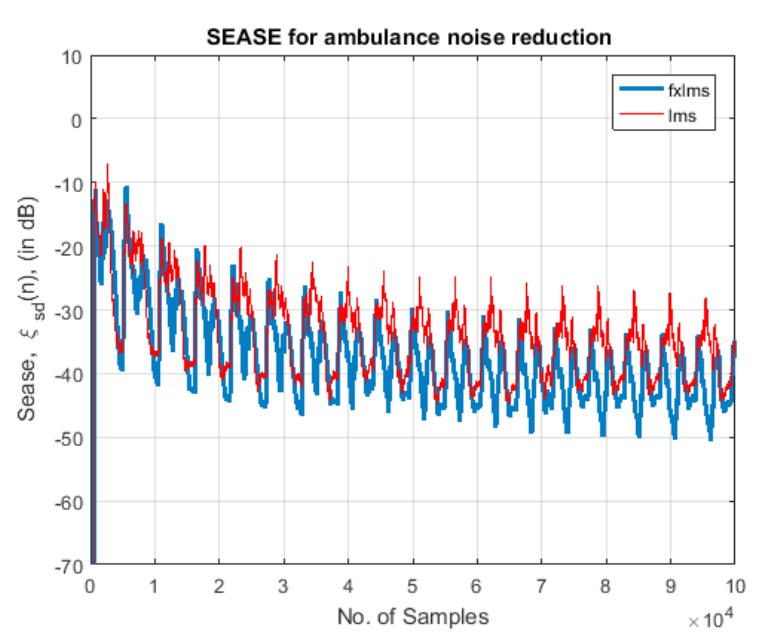

Figure 8. SEASE comparison using LMS and FxLMS algorithms.

The plots of power spectral density (PSD) of the reference signal, error signal using LMS and FxLMS algorithms are shown in Figure 7. The results obtained analytically are verified with PSD graph in Figure 7.

The simulations are performed many times using different step sizes for both the algorithms.

The average of errors obtained for simulations done using different step sizes is evaluated by smoothened ensemble average square error (SEASE), $\xi(n)$. SEASE is calculated as per the equations (8) to (10):

$$
\begin{aligned}
& \xi(\mathrm{n})=\frac{1}{\mathrm{~K}} \sum_{\mathrm{i}=1}^{\mathrm{K}} \mathrm{e}_{\mathrm{i}}^{2}(\mathrm{n}) \\
& \xi_{\mathrm{s}}(\mathrm{n})=\sigma \xi_{\mathrm{s}}(\mathrm{n}-1)+(1-\sigma) \xi(\mathrm{n}), 0<\sigma<1 \\
& \xi_{\mathrm{Sd}}(\mathrm{n})=10 \log _{10}\left(\xi_{\mathrm{s}}(\mathrm{n})\right)
\end{aligned}
$$

where, $K$ represents number of trials,

$e_{i}(n)$ is error of the $i$ th trial

$\xi_{S N}$ is normalized value of $\xi_{S d}$

The comparison of SEASE for LMS and FxLMS algorithm based ANC system is shown in Figure 8. It is observed that performance of active noise control with FxLMS for ambulance siren gives better results than LMS with a difference in noise reduction of more than $3 \mathrm{~dB}$.

\section{Conclusion}

It is matter of concern that noise from ambulance siren is a reason for deteriorating the condition of patient lying inside the ambulance. The ANC technique has advantage over passive noise technique in reducing the noise. This paper is focused on the use of ANC system for reducing noise generated from ambulance siren. The analyses are done in MATLAB based on LMS and FxLMS adaptive algorithms and the results are compared. The simulations show a noise reduction of approximately $25 \mathrm{~dB}$ from LMS algorithm and $28 \mathrm{~dB}$ from FxLMS algorithm. This shows the supremacy of FxLMS algorithm over LMS algorithm in reducing noise more efficiently and it provides more stable system as the practical components such as ADC, $\mathrm{DAC}$, etc. used in real time application are taken into consideration. This indicates that FxLMS will be robust algorithm which can be implemented in real time for various applications.

\section{Acknowledgements}

The work reported in this article is supported by University Grants Commission (UGC), New Delhi.

\section{References}

1. Hearing conservation. Date accessed 20/06/15: Available from: http://ehs.unl.edu/sop/s-hearing_conservation.pdf.

2. Weber U, Reitinger A, Szusz R, Hellmich C, Steinlechner B, Hager H, Mora B, Selzer M, Hiesmayr M, Kober A. Emergency ambulance transport induces stress in patients with acute coronary syndrome. Emergency Medicine Journal. 2009 Jul 1; 26(7):524-28.

3. Corliss EL, Jones FE. Method for estimating the audibility and effective loudness of sirens and speech in automobiles. The Journal of the Acoustical Society of America. 1976 Nov $1 ; 60(5): 1126-31$.

4. Howard CQ, Maddern AJ, Privopoulos EP. Acoustic characteristics for effective ambulance sirens. Acoustics Australia. 2011 August; 39(2):43-53.

5. Elliott SJ, Nelson PA. Active noise control. Signal Processing Magazine, IEEE. 1993 Oct; 10(4):12-35.

6. Kuo SM, Morgan DR. New York: Wiley: Active Noise Control Systems - Algorithms and DSP Implementations. 1996.

7. Elliott S. Signal processing for active control. Academic press: 2000. 
8. Strauch P, Mulgrew B. Active control of nonlinear noise processes in a linear duct. IEEE Transactions on Signal Processing. 1998 September; 46(9):2404-12.

9. Kuo SM, Morgan DR. Active noise control: a tutorial review. Proceedings of the IEEE. 1999 Jun; 87(6):943-73.

10. Elliott SJ, Nelson PA. The application of adaptive filtering to the active control of sound and vibration. NASA STI/Recon Technical Report N. 1985 Sep; 86:326-28.

11. Morgan DR. An analysis of multiple correlation cancellation loops with a filter in the auxiliary path. IEEE Transactions on Acoustics, Speech and Signal Processing. 1980 August; 28(4):454-67.

12. Kajikawa Y, Gan WS, Kuo SM. Recent advances on active noise control: open issues and innovative applications.
APSIPA Transactions on Signal and Information Processing. 2012 Dec; 1:e3.

13. Burgess JC. Active adaptive sound control in a duct: A computer simulation. The Journal of the Acoustical Society of America. 1981 September 1; 70(3):715-26.

14. Widrow B, Stearns SD. Englewood Cliffs, NJ, Prentice-Hall, Inc.: Adaptive Signal Processing. 1985; 491:1985;1.

15. Sharma MK, Vig R. Reduction in Acoustic Noise Generated from Ambulance Siren Using Active Noise Control. Chandigarh, India: Proceedings of 3rd International Conference on Biomedical Engineering and Assistive Technologies. 2014; p. 265-268. 